\title{
On the duality relation for correlation functions of the Potts model
}

\author{
Wentao T. Lu and F. Y. Wu \\ Department of Physics \\ Northeastern University, Boston, Massachusetts 02115
}

\begin{abstract}
We prove a recent conjecture on the duality relation for correlation functions of the Potts model for boundary spins of a planar lattice. Specifically, we deduce the explicit expression for the duality of the $n$-site correlation functions, and establish sum rule identities in the form of the Möbius inversion of a partially ordered set. The strategy of the proof is by first formulating the problem for the more general chiral Potts model. The extension of our consideration to the many-component Potts models is also given.
\end{abstract}

$05.50 .+\mathrm{q}$ 


\section{INTRODUCTION}

The duality relation for the $q$-state Potts model [1,2] is an identity [3] relating the partition functions of a Potts model on a planar lattice with that of its dual. Very recently, this duality consideration has been extended to the Potts correlation functions [4,5]. Specifically, it was established that certain duality relations exist for correlation functions of $n$ Potts spins on the boundary of a planar lattice. Explicit expressions for the duality relation have been obtained for $n=2,3$ [四] and $n=4$ [5]; the expression for general $n$ has also been conjectured [5]. It has also been shown that there exist certain sum rule identities, but the explicit form of the identities was not given [5]. The purpose of this paper is the following: We first extend the consideration to the chiral Potts model [6] and obtain its duality relation in a very general form. We then show that this formulation permits us to establish the conjecture of [5]. In addition, it also leads to an expression of the sum rule identities in the form of the Möbius inversion of a partially ordered set. Furthermore, starting from a multi-component chiral Potts model [7], we extend considerations to the multi-component Potts model, which includes the Ashkin-Teller model [9] and its generalization [10]. This extends results reported elsewhere [11] for the 2-component Potts model.

\section{THE CHIRAL POTTS MODEL}

Consider a $q$-state spin system on a two-dimensional lattice or, more generally, any planar graph $\mathcal{L}$. Let $x_{i}=1,2, \cdots, q$ denote the spin state of the $i$-th site and $-J\left(x_{i}, x_{j}\right)$ the interaction energy between sites $i$ and $j$, which can be edge-dependent. The interaction is chiral if

$$
J\left(x, x^{\prime}\right) \neq J\left(x^{\prime}, x\right) .
$$

The partition function of this spin system is

$$
Z(u)=\sum_{x_{i}=1}^{q} \prod_{<i j>} U\left(x_{i}, x_{j}\right),
$$

where $U\left(x, x^{\prime}\right)=\exp \left[J\left(x, x^{\prime}\right) / k T\right]$ and the product is taken over all edges of $\mathcal{L}$.

For our purposes we consider the chiral Potts model [6] for which the Boltzmann factor is cyclic, namely, it satisfies

$$
U\left(x, x^{\prime}\right)=u\left(x-x^{\prime}\right), \quad(\bmod q) .
$$

We shall refer to the $q \times q$ matrix $\mathbf{U}$ as the interaction matrix. The chiral Potts model reduces to the standard Potts model upon taking $J\left(x, x^{\prime}\right) / k T=K \delta\left(x, x^{\prime}\right)$, where $\delta$ is the Kronecker delta function which we define as

$$
\begin{array}{rlrl}
\delta\left(x, x^{\prime}\right) & =1, & & x=x^{\prime} \\
& =0, & & (\bmod q) \\
& =x^{\prime} & (\bmod q) .
\end{array}
$$

This leads to 


$$
u(x)=1+\left(e^{K}-1\right) \delta(x, 0) .
$$

Duality relation for the partition function: The partition function of the chiral Potts model possesses a duality relation [3]. Let $\mathcal{L}^{D}$ be the dual of $\mathcal{L}$, and let $Z^{(D)}\left(u^{*}\right)$ be the partition function of the chiral Potts model on $\mathcal{L}^{D}$ with the Boltzmann factor

$$
u^{*}(y)=\frac{1}{\sqrt{q}} \sum_{x=1}^{q} \omega^{x y} u(x), \quad y=1,2, \cdots, q
$$

where $\omega=e^{2 \pi i / q}$. Then, the duality relation reads [3]

$$
q^{-N / 2} Z[u(x)]=q^{-N^{*} / 2} Z^{(D)}\left[u^{*}(y)\right],
$$

where $N$ and $N^{*}$ are, respectively, the numbers of sites of $\mathcal{L}$ and $\mathcal{L}^{D}$. They are related to the number of edges $\mathrm{E}$ by the Euler relation $N+N^{*}=E+2$. This duality relation holds for arbitrary edge-dependent interactions. Note that $\sqrt{q} u^{*}(y), y=1, \cdots, q$, are the eigenvalues of the matrix $\mathbf{U}$. In the case of the standard Potts model, (6) reduces to

$$
u^{*}(y)=\frac{1}{\sqrt{q}}\left[e^{K}-1+q \delta(y, 0)\right] .
$$

\section{DUALITY RELATION FOR THE CORRELATION FUNCTION}

Number $n$ sites on the boundary of $\mathcal{L}$ by integers $1,2, \cdots n$ clockwise as shown in Fig. 1. The probability that the $n$ sites are in respective spin states $x_{1}, x_{2}, \cdots, x_{n}$ is

$$
P_{n}\left(x_{1}, x_{2}, \cdots, x_{n}\right)=Z_{x_{1} x_{2} \cdots x_{n}} / Z(u),
$$

where $Z_{x_{1} x_{2} \cdots x_{n}}$ is the partial partition function of the chiral Potts model with the $n$ spins in fixed given states. Construct an auxiliary lattice $\mathcal{L}_{\text {aux }}$ from $\mathcal{L}$ by connecting all $n$ boundary sites to an extra site $e$ in the infinite face of $\mathcal{L}$ as shown. These $n$ connecting lines then divide the infinite face of $\mathcal{L}$ into $n$ parts, which we also number by $1,2, \cdots, n$ as shown in Fig. 1. Let $u_{j e}, j=1,2, \cdots n$, be the Boltzmann factor of the line connecting sites $j$ to $e$. Its dual edge then connects sites (residing in faces of $\mathcal{L}$ ) $j$ and $j+1$ on $\mathcal{L}_{\text {aux }}^{D}$ with the Boltzmann factor

$$
u_{j+1, j}^{*}\left(y_{j+1}-y_{j}\right)=\frac{1}{\sqrt{q}} \sum_{x=1}^{q} \omega^{\left(y_{j+1}-y_{j}\right) x} u_{j e}(x), \quad j=1, \cdots, n
$$

where $y_{j}$ denotes the spin state of the dual site $j$. Here we have adopted the convention of [3] in orienting the edges. It is also convenient to consider the $n$-spin correlation [4]

\footnotetext{
${ }^{1}$ The definition of the dual Boltzmann factor adopted here differs from that used in [3] by a factor $\sqrt{q}$. The present definition is consistent to the notion of $\left(u^{*}\right)^{*}=u$.
} 


$$
\Gamma_{n}=q^{n} P_{n}(x, x, \cdots, x)-1,
$$

a quantity which vanishes identically if the $n$ spins are completely uncorrelated.

We apply the duality relation $(7)$ to $\mathcal{L}_{\text {aux }}$. Let $Z_{y_{1} y_{2} \cdots y_{n}}^{*}$ be the partial partition function of the Potts model on $\mathcal{L}_{\text {aux }}^{D}$ with sites $1,2, \cdots, n$ in respective states $y_{1}, y_{2}, \cdots, y_{n}$. Then, explicitly, (Ø) reads

$$
\begin{aligned}
q^{-(N+1) / 2} \sum_{x_{1} x_{2} \cdots x_{n}} \sum_{x_{e}} u_{1 e}\left(x_{1 e}\right) u_{2 e}\left(x_{2 e}\right) \cdots u_{n e}\left(x_{n e}\right) Z_{x_{1} x_{2} \cdots x_{n}} \\
=q^{-\left(N^{*}+n-1\right) / 2} \sum_{y_{1} y_{2} \cdots y_{n}} u_{21}^{*}\left(y_{21}\right) u_{32}^{*}\left(y_{32}\right) \cdots u_{1 n}^{*}\left(y_{1 n}\right) Z_{y_{1} y_{2} \cdots y_{n}}^{*}
\end{aligned}
$$

where $x_{1 e}=x_{1}-x_{e}, y_{21}=y_{2}-y_{1}$, etc., and we have used the fact that $\mathcal{L}_{\text {aux }}$ has $N+1$ sites and $\mathcal{L}_{\text {aux }}^{D}$ has $N^{*}+n-1$ sites.'t]

Due to the cyclic nature of the matrix elements, we can replace the arguments $x_{1 e}, x_{2 e}, \cdots$ on the LHS of (12) by $x_{1}, x_{2}, \cdots$. The summation over $x_{e}$ can then be carried out leading to an overall factor $q$. Next we eliminate $u^{*}$ by using (10). Since all $u$ factors in (12) are independent, as a consequence we can equate the coefficients of $u_{1 e}\left(x_{1}\right) u_{2 e}\left(x_{2}\right) \cdots u_{n e}\left(x_{n}\right)$ in (12). This leads to the duality identity

$$
Z_{x_{1} x_{2} \cdots x_{n}}=C_{n}(q) \sum_{y_{1} y_{2} \cdots y_{n}} M\left(x_{1}, x_{2}, \cdots, x_{n} \mid y_{1}, y_{2}, \cdots, y_{n}\right) Z_{y_{1} y_{2} \cdots y_{n}}^{*}
$$

where

$$
C_{n}(q)=q^{-n+\left(N-N^{*}\right) / 2},
$$

and

$$
M\left(x_{1}, x_{2}, \cdots, x_{n} \mid y_{1}, y_{2}, \cdots, y_{n}\right)=\omega^{x_{n 1} y_{1}+x_{12} y_{2}+\cdots+x_{(n-1) n} y_{n}}
$$

can be interpreted as the elements of a $q^{n} \times q^{n}$ matrix M. Eq. (13) is the duality relation for the chiral Potts model, and is a very general expression. The relation assumes a reduced form for the standard Potts model after taking into account the degeneracy of states, and the crux of matter is, of course, to deduce the reduced relation. This is the subject matter of the next section.

Introducing the notation

$$
p_{y_{1} y_{2} \cdots y_{n}} \equiv Z_{y_{1} y_{2} \cdots y_{n}}^{*} / Z_{11 \cdots 1}^{*}=q Z_{y_{1} y_{2} \cdots y_{n}}^{*} / Z^{(D)}
$$

and combining with (7) and (9), we can rewrite (13) as

$$
P_{n}\left(x_{1}, x_{2}, \cdots, x_{n}\right)=q^{-(n+1)} \sum_{y_{1} y_{2} \cdots y_{n}} M\left(x_{1}, x_{2}, \cdots, x_{n-1}, x_{n} \mid y_{1}, y_{2}, \cdots, y_{n}\right) p_{y_{1} y_{2} \cdots y_{n}} .
$$

An immediate consequence of (17) is

\footnotetext{
${ }^{2}$ Note that the partial partition function $Z_{y_{1} y_{2} \cdots y_{n}}^{*}$ in (12) differs from that in 4 . 5 ] by a factor.
} 


$$
\Gamma_{n}=\frac{1}{q} \sum_{y_{1} y_{2} \cdots y_{n}} p_{y_{1} y_{2} \cdots y_{n}}-1,
$$

a result which has been reported previously [5, 12].

To obtain the inverse of (13), we note that, by deleting the $\{1,2\},\{2,3\}, \cdots$ edges in

$\mathcal{L}_{\text {aux }}^{D}$, we arrive at a lattice $\mathcal{L}^{*}$ in which $1,2, \cdots$ of $\mathcal{L}^{*}$ are its boundary sites. An example of $\mathcal{L}$ and the resulting $\mathcal{L}^{*}$ is shown in Fig. 2. It is clear that the roles of $\mathcal{L}$ and $\mathcal{L}^{*}$ are reciprocal, so we can apply (13i) to $\mathcal{L}^{*}$ to obtain an inverse transform. Now $\mathcal{L}^{*}$ has $\bar{N}=N^{*}+n-1$ sites and its dual has $\bar{N}^{*}=N-n+1$ sites. As a result, we obtain

$$
Z_{y_{1} y_{2} \cdots y_{n}}^{*}=\bar{c}_{n}(q) \sum_{x_{1} x_{2} \cdots x_{n}} M\left(y_{1}, y_{2}, \cdots, y_{n} \mid x_{n}, x_{1}, x_{2}, \cdots, x_{n-1}\right) Z_{x_{1} x_{2} \cdots x_{n}},
$$

where

$$
\bar{c}_{n}(q)=q^{-n+\left(\bar{N}-\bar{N}^{*}\right) / 2}=q^{-1+\left(N^{*}-N\right) / 2} .
$$

We have $c_{n}(q) \bar{c}_{n}(q)=q^{-(n+1)}$ and note that the sequence $\left\{x_{n}, x_{1}, x_{2}, \cdots, x_{n-1}\right\}$ of the indices on the RHS is related to $\left\{x_{1}, x_{2}, \cdots, x_{n-1}, x_{n}\right\}$ by a simple cyclic permutation. Upon combining (19) with (13) we obtain the identity

$$
\mathbf{M}^{2}\left(x_{1}, x_{2}, \cdots, x_{n} \mid x_{1}^{\prime}, x_{2}^{\prime}, \cdots, x_{n}^{\prime}\right)=q^{n+1} \delta\left(x_{1}, x_{2}^{\prime}\right) \delta\left(x_{2}, x_{3}^{\prime}\right) \cdots \delta\left(x_{n}, x_{1}^{\prime}\right),
$$

which we refer to as a reciprocal inversion relation.

\section{THE STANDARD POTTS MODEL}

For the standard Potts model the duality relation (13) assumes a reduced form which has been given previously for $n=2,3$ [4] and for $n=4$ [5]. The form for general $n$ has also been conjectured [5]. It has also been shown that the correlation functions satisfy certain sum-rule identities [5]. Here, we reformulate the standard Potts model as an instance of the chiral Potts model and, as we shall see, this leads us to establish the conjecture as well as deduce the general expression for the sum rule identities. For this reason, it is instructive to first demonstrate how (13) reduces to the known results for $n=2,3$.

The standard Potts model is characterized by the fact that one needs only to keep track of spin states that are the same. That is to say, one needs to keep track of the partition of the $n$ sites into blocks, such that sites in one block are in the same state. This leads to writing the partial partition functions in an expansion of a partially ordered set. Along this line, we writes for $n=2,3$,

$$
\begin{aligned}
Z_{x_{1} x_{2}} & =D_{12}+D_{11} \delta\left(x_{1}, x_{2}\right) \\
Z_{y_{1} y_{2}}^{*} & =D_{12}^{*}+D_{11}^{*} \delta\left(y_{1}, y_{2}\right) \\
Z_{x_{1} x_{2} x_{3}} & =D_{123}+D_{113} \delta\left(x_{1}, x_{2}\right)+D_{121} \delta\left(x_{1}, x_{3}\right)+D_{122} \delta\left(x_{2}, x_{3}\right)+D_{111} \delta\left(x_{1}, x_{2}, x_{3}\right) \\
Z_{y_{1} y_{2} y_{3}}^{*} & =D_{123}^{*}+D_{113}^{*} \delta\left(y_{1}, y_{2}\right)+D_{121}^{*} \delta\left(y_{1}, y_{3}\right)+D_{122}^{*} \delta\left(y_{2}, y_{3}\right)+D_{111}^{*} \delta\left(y_{1}, y_{2}, y_{3}\right),
\end{aligned}
$$

where $\delta\left(x_{1}, x_{2}, x_{3}\right)=\delta\left(x_{1}, x_{2}\right) \delta\left(x_{2}, x_{3}\right)$. Here, for $n=3$, for example, the partition of the 3 -set of integers $\{1,2,3\}$ includes the 5 elements $\{\{1\},\{2\},\{3\}\},\{\{12\},\{3\}\},\{\{1\},\{23\}\}$, $\{\{13\},\{2\}\},\{\{123\}\}$ denoted by subscripts $\{123\},\{113\},\{122\},\{121\},\{111\}$, respectively. 
For $n=2$, we substitute the first two lines of (22) into (13). Making use of the identity

$$
\sum_{y=1}^{q} \omega^{x y}=q \delta(x, 0),
$$

one arrives at

$$
\begin{aligned}
D_{12}+D_{11} \delta\left(x_{1}, x_{2}\right) & =C_{2}(q) \sum_{y_{1} y_{2}} \omega^{x_{21} y_{1}+x_{12} y_{2}}\left[D_{12}^{*}+D_{11}^{*} \delta\left(y_{1}, y_{2}\right)\right] \\
& =C_{2}(q)\left[q^{2} D_{12}^{*} \delta\left(x_{1}, x_{2}\right)+q D_{11}^{*}\right] .
\end{aligned}
$$

Since this equation holds for arbitrary $x_{1}$ and $x_{2}$, the coefficients of corresponding delta functions must be equal, and this leads to the identities

$$
D_{12}=C_{2}(q) q D_{11}^{*}, \quad D_{11}=C_{2}(q) q^{2} D_{12}^{*},
$$

which can further be converted to relate $Z_{x_{1} x_{2}}$ to $Z_{x_{1}, x_{2}}^{*}$ as follows. First, the second line of (22) gives

$$
Z_{12}^{*}=D_{12}^{*}, \quad Z_{11}^{*}=D_{12}^{*}+D_{11}^{*},
$$

from which one obtains the inverse

$$
D_{12}^{*}=Z_{12}^{*}, \quad D_{11}^{*}=Z_{11}^{*}-Z_{12}^{*} .
$$

Substituting (27) into (25) and combining (9), (7) and the definition (16), one is led to the duality relation for the 2-point correlation function

$$
\begin{aligned}
P_{2}\left(x_{1}, x_{2}\right) & =\left[D_{12}+D_{11} \delta\left(x_{1}, x_{2}\right)\right] / Z \\
& =\frac{1}{q^{2}}\left[1+\left(q \delta\left(x_{1}, x_{2}\right)-1\right) p_{12}\right] .
\end{aligned}
$$

This is the duality relation (17) for $n=2$. Finally, using (22), (25), and (27), and introducing the row vector $\tilde{\mathbf{z}}_{2}=\left(Z_{11}, Z_{12}\right)$, $\tilde{\mathbf{z}}_{2}^{*}=\left(Z_{11}^{*}, Z_{12}^{*}\right)$, we find

$$
\mathbf{z}_{2}=q C_{2}(q) \mathbf{T}_{2}(q) \cdot \mathbf{z}_{2}^{*}
$$

where

$$
\mathbf{T}_{2}(q)=\left(\begin{array}{cc}
1 & q-1 \\
1 & -1
\end{array}\right)
$$

satisfying $\left[\mathbf{T}_{2}(q)\right]^{2}=q \mathbf{I}_{2}$, with $\mathbf{I}_{2}$ being the $2 \times 2$ identity matrix.

Similarly for $n=3$ one obtains in place of (24)

$$
\begin{aligned}
& D_{123}+D_{113} \delta\left(x_{1}, x_{2}\right)+D_{121} \delta\left(x_{1}, x_{3}\right)+D_{122} \delta\left(x_{2}, x_{3}\right)+D_{111} \delta\left(x_{1}, x_{2}, x_{3}\right) \\
& =C_{3}(q) \sum_{y_{1} y_{2} y_{3}} \omega^{x_{31} y_{1}+x_{12} y_{2}+x_{23} y_{3}}\left[D_{123}^{*}+D_{113}^{*} \delta\left(y_{1}, y_{2}\right)+D_{121}^{*} \delta\left(y_{1}, y_{3}\right)\right. \\
& \left.+D_{122}^{*} \delta\left(y_{2}, y_{3}\right)+D_{111}^{*} \delta\left(y_{1}, y_{2}, y_{3}\right)\right]
\end{aligned}
$$


leading to the identities

$$
\begin{aligned}
& D_{123}=C_{3}(q) q D_{111}^{*}, \quad D_{111}=C_{3}(q) q^{3} D_{123}^{*} \\
& D_{113}=C_{3} q^{2} D_{121}^{*}, \quad D_{121}=C_{3}(q) q^{2} D_{122}^{*}, \quad D_{122}=C_{3}(q) q^{2} D_{113}^{*} .
\end{aligned}
$$

Again, we can rewrite (32) in a form relating $Z_{x_{1} x_{2} x_{3}}$ to $Z_{y_{1} y_{2} y_{3}}^{*}$. Now the inverse of the last line of (22) is

$$
\begin{aligned}
& D_{123}^{*}=Z_{123}^{*} \\
& D_{113}^{*}=Z_{113}^{*}-Z_{123}^{*}, \quad D_{121}^{*}=Z_{121}^{*}-Z_{123}^{*}, \quad D_{122}^{*}=Z_{122}^{*}-Z_{123}^{*}, \\
& D_{111}^{*}=Z_{111}^{*}-\left(Z_{113}^{*}+Z_{121}^{*}+Z_{122}^{*}\right)+2 Z_{123}^{*} .
\end{aligned}
$$

Substituting (33) into (32) and using the third line of (22) for $Z_{x_{1} x_{2} x_{3}}$, we obtain

$$
\begin{aligned}
P_{3}\left(x_{1}, x_{2}, x_{3}\right)= & \frac{1}{q^{3}}\left[1-\left(p_{122}+p_{121}+p_{113}\right)+2 p_{123}+q\left(p_{121}-p_{123}\right) \delta\left(x_{1}, x_{2}\right)\right. \\
& \left.+q\left(p_{122}-p_{123}\right) \delta\left(x_{1}, x_{3}\right)+q\left(p_{113}-p_{123}\right) \delta\left(x_{2}, x_{3}\right)+q^{2} p_{123} \delta\left(x_{1}, x_{2}, x_{3}\right)\right] .
\end{aligned}
$$

This is the duality relation (17) for $n=3$ reported in [4].

Explicitly, introducing the row vector $\tilde{\mathbf{z}}_{3}=\left(Z_{111}, Z_{113}, Z_{121}, Z_{122}, Z_{123}\right)$ and similarly defined $\tilde{\mathbf{z}}_{3}^{*}$, we find

$$
\mathbf{z}_{3}=q C_{3}(q) \mathbf{T}_{3}(q) \cdot \mathbf{z}_{3}^{*}
$$

where

$$
\mathbf{T}_{3}(q)=\left(\begin{array}{ccccc}
1 & q-1 & q-1 & q-1 & (q-1)(q-2) \\
1 & -1 & q-1 & -1 & -(q-2) \\
1 & -1 & -1 & q-1 & -(q-2) \\
1 & q-1 & -1 & -1 & -(q-2) \\
1 & -1 & -1 & -1 & 2
\end{array}\right)
$$

satisfying

$$
\left[\mathbf{T}_{3}(q)\right]^{2}=q^{2}\left(\begin{array}{ccccc}
1 & 0 & 0 & 0 & 0 \\
0 & 0 & 0 & 1 & 0 \\
0 & 1 & 0 & 0 & 0 \\
0 & 0 & 1 & 0 & 0 \\
0 & 0 & 0 & 0 & 1
\end{array}\right)
$$

\footnotetext{
${ }^{3}$ Note, however, the numbering of $\left\{y_{1}, y_{2}, y_{3}\right\}$ as $\left\{s^{\prime}, s^{\prime \prime}, s\right\}$ in [4], resulting in a more symmetric appearance of (34).
} 


\section{THE GENERAL ANALYSIS}

For general $n$, we write $Z_{x_{1} \cdots x_{n}}$ as a sum in the form of (22), namely,

$$
Z_{x_{1} \cdots x_{n}}=\sum_{X} D_{X} \delta(X)
$$

where the summation is over all partitions $X$ of $n$ integers $\{1,2, \cdots, n\}$, and $\delta(X)$ is a product of delta functions associated with blocks in $X$. For example, we have $\delta(X)=$ $\delta\left(x_{1}, x_{2}\right) \delta\left(x_{3}, x_{4}\right)$ for $X=\{1133\}$, and $\delta(X)=\delta\left(x_{1}, x_{2}, x_{3}\right)=\delta\left(x_{1}, x_{2}\right) \delta\left(x_{2}, x_{3}\right)$ for $X=$ $\{1114\}$. If a block of $X$ contains $m \geq 3 x$ 's as in $\delta\left(x_{1}, x_{2}, x_{3}\right)$ above, we write $\delta(X)$ as a product of $m-12$-point delta functions $\delta\left(x_{j}, x_{k}\right)$ with the $x$ 's arranged in, say, a clockwise sequence, so the writing of $\delta(X)$ as a product of 2-point delta functions is unique. The number of terms in (38) is $b_{n}$, and it has been shown in [5] that $b_{n}$ is generated by

$$
\sum_{n=0}^{\infty} b_{n} t^{n} / n !=\exp \left(e^{t}-1\right) .
$$

Without the loss of generality we shall assume $q \geq n$ so that all $Z_{X}$ are physically realized.'

The partition sums (22) and (38) can be regarded as a transformation between the $Z$ 's and $D$ 's. The transformation is that of a partially ordered set and it is known [13 that the inverse is given by the Möbius inversion. Let $X, X^{\prime}, Y$, etc. denote specific partitions of an $n$-set. We write $X^{\prime} \preceq X$ if the block structure of $X^{\prime}$ is contained in $X$, namely, $X^{\prime}$ is a refinement of $X$ [13]. For example, $\{1134\}$ and $\{1234\}$ are contained in $\{1133\}$, while $\{1224\}$ is not. Then, an immediate consequence of (38) is 13.

$$
Z_{X}=\sum_{X^{\prime} \preceq X} D_{X^{\prime}}
$$

In addition, the Möbius inversion of $(38)$ is

$$
D_{X}=\sum_{X^{\prime} \preceq X} \mu\left(X^{\prime}, X\right) Z_{X^{\prime}}
$$

with

$$
\mu\left(X^{\prime}, X\right)=(-1)^{\left|X^{\prime}\right|-|X|} \prod_{\text {blocks } \in X}\left(n_{b}-1\right) !
$$

where $|X|$ and $\left|X^{\prime}\right|$ are, respectively, the number of blocks in $X$ and $X^{\prime}$, and $n_{b}$ the number of blocks of $X^{\prime}$ that are contained in a block of $X$. In the examples above, for instance, $X=\{1133\}$ has two blocks, and we have $n_{b}=\{1,2\}$ for $X^{\prime}=\{1134\}$, and $n_{b}=\{2,2\}$ for $X^{\prime}=\{1234\}$, etc. This leads to

\footnotetext{
${ }^{4}$ Otherwise the situation $q<|X| \leq n$ can occur, where $|X|$ is the number of blocks of the partition $X$, and $Z_{X}$ has no physical meaning. But $Z_{X}$, which can be computed for $q \geq|X|$, is a polynomial in $q$. We shall take $Z_{X}$ to be the same polynomial for all $q$ including $q<|X|$. With this understanding in mind all our results, including the identities derived below, hold for all $q, n$.
} 


$$
\begin{aligned}
D_{1133} & =(-1)^{2-2}(0 !)^{2} Z_{1133}+(-1)^{3-2}(0 !)(1 !) Z_{1134}+(-1)^{3-2}(1 !)(0 !) Z_{1233}+(-1)^{4-2}(1 !)^{2} Z_{1234} \\
& =Z_{1133}-Z_{1134}-Z_{1233}+Z_{1234} .
\end{aligned}
$$

Other examples are

$$
\begin{aligned}
& D_{1114}=Z_{1114}-Z_{1134}-Z_{1214}-Z_{1224}+2 Z_{1234} \\
& D_{1212}=Z_{1212}-Z_{1214}-Z_{1232}+Z_{1234} .
\end{aligned}
$$

Substitute the partition sum (38) and a similar expression for $Z_{y_{1} \cdots y_{n}}^{*}$ into (13), we obtain

$$
\sum_{X} D_{X} \delta(X)=C_{n}(q) \sum_{Y} \sum_{y_{1} \cdots y_{n}} M\left(x_{1}, \cdots x_{n} \mid y_{1}, \cdots y_{n}\right) D_{Y}^{*} \delta(Y) .
$$

It turns out that the analysis is best done graphically. Represent the matrix element $M\left(x_{1}, \cdots, x_{n} \mid y_{1}, \cdots, y_{n}\right)$ in (13) by a graph $G_{n}$ shown in Fig. 3, where each node (open circle) denotes a $y$-summation, and each arc is assigned a label $x$ such that an outgoing arrow (from a node labelled $y$ ) carries a factor $\omega^{-x y}$ and an incoming arrow a factor $\omega^{x y}$. To each $X$ we construct a "connectivity" $\Gamma_{X}$ according to the following prescription: Connect the mid-points of the arcs belonging to each block of $X$ to a common point exterior to $G_{n}$. A connectivity is planar if the connecting lines do not intersect; otherwise the connectivity is non-planar. Examples of connectivities for $G_{4}$ are shown in Fig. 4. We note that the construction of the connectivities is the same as that introduced in [5]. We have two possibilities.

i) $\Gamma_{X}$ is planar. In this case $\Gamma_{X}$ divides the region exterior to $G_{n}$ into faces. Regarding all nodes in one face as belonging to a block of another partition $Y$, and denote this mapping by $X \rightarrow Y$. It is clear that $\Gamma_{Y}$ is also planar and that $Y \rightarrow X$. Therefore the mapping $X \leftrightarrow Y$ is one-one. One has alsof

$$
|X|+|Y|=n+1
$$

It has been shown [14,15] that the number $c_{n}$ of planar $\Gamma_{X}$, or simply planar $X$, is generated by

$$
\sum_{n=0}^{\infty} c_{n} t^{n}=(1-\sqrt{1-4 t}) / 2 t .
$$

With these understandings, we now carry out the $y$-summations on the RHS of (45). From (23) each $y$-summation yields a factor $q \delta\left(x, x^{\prime}\right)$, where $x$ and $x^{\prime}$ are the labellings of the incoming and outgoing arrows. One can then regard the labellings $x$ as a flow which is conserved at each node. Further, the effect of the delta function $\delta(Y)$ in (45) is to collapse the $y$-summations of each block of $Y$ into a single summation, resulting in a factor $q \delta\left(x_{\mathrm{in}}, x_{\mathrm{out}}\right)$ where $x_{\text {in }}$ is the sum of the incoming flows and $x_{\text {out }}$ the sum of outgoing flows. This effect

\footnotetext{
${ }^{5}$ For $X=\left\{\left\{x_{1}\right\},\left\{x_{2}\right\}, \cdots,\left\{x_{n}\right\}\right\},|X|=n$, we have $Y=\left\{\left\{y_{1}, y_{2}, \cdots, y_{n}\right\}\right\},|Y|=1$. It is also clear that $|Y|$ increases by 1 when $|X|$ decreases by 1 . This establishes (46).
} 
can be conveniently visualized by contracting the nodes in question into a single one, and requiring that the flow is conserved at the contracted node. For example, as shown in Fig. 5, the partition $Y=\{123216\}$ of $G_{6}$ is contracted into a diagram consisting of a sequence of $3 G_{2}$ "cactus leaves" attached together at two common nodes. Generally, the delta function $\delta(Y)$ contracts $G_{n}$ into a diagram consisting of cactus leaves $G_{m}, m<n$, attached together at common nodes. The planar nature of $Y$ then ensures that the resulting diagram is a cactus tree. One next carries out the $y$-summations one-by-one starting from the nodes of the outermost leaves of the tree. The tree structure now ensures that this always leads to a product of 2-point delta functions. In the example shown in Fig. 5, for instance, this leads to

$$
\delta\left(x_{5}, x_{6}\right) \delta\left(x_{4}+x_{6}, x_{1}+x_{5}\right) \delta\left(x_{1}+x_{3}, x_{2}+x_{4}\right) \delta\left(x_{2}, x_{3}\right)=\delta\left(x_{5}, x_{6}\right) \delta\left(x_{4}, x_{1}\right) \delta\left(x_{2}, x_{3}\right) .
$$

But this is precisely the factor $\delta(X)$ appearing in $D_{X} \delta(X)$ on the LHS of (45), where $X=\{122155\} \leftrightarrow Y$. It is readily verified that, generally for each planar $X \leftrightarrow Y$, to the term $D_{X} \delta(X)$ on the LHS of (45), the $y$-summations of the $Y$ term on the RHS yields a factor $D_{Y} q^{|Y|} \delta(X)$. Now, the indices $\left\{x_{i}\right\}$ in a given $\delta(X)$ are arbitrary. It follows that the coefficients of the two terms must be equal, and this leads to the identity

$$
D_{X}=C_{n}(q) q^{|Y|} D_{Y}^{*}, \quad \text { planar } X \leftrightarrow Y,
$$

an equation which generalizes (25) and (32).

ii) $\Gamma_{X}$ is non-planar. We now consider the remaining $b_{n}-c_{n}$ terms in (45). Now, each remaining terms on the LHS is of the form $D_{X} \delta(X)$, where $\delta(X)$ is a product of 2-point delta functions $\delta\left(x_{j}, x_{k}\right)$. For the remaining terms on the RHS we again carry out the $y$ summations for each $Y$. However, due to the fact that $Y$ is now non-planar, the process of contraction as described in the above leads to diagrams in which cactus leaves form rings, or circuits. It follows that the $y$-summations generates a product involving some 4 -point delta functions of the type $\delta\left(x_{i}+x_{j}, x_{k}+x_{\ell}\right)$, which cannot be reduced into products of 2-point delta functions as in (48). In other words, the delta functions $\delta(X)$ occurring on the LHS do not appear on the RHS, and vice versa. Now, the the indices $\left\{x_{i}\right\}$ in a given $\delta(X)$ are arbitrary. It follows that, for the equality (45) to hold for all $\left\{x_{i}\right\}$, each of these remaining coefficients must vanish individually. Namely, we must have

$$
D_{X}=D_{Y}^{*}=0, \quad \text { non }- \text { planar } X, Y .
$$

Finally introduce the partially ordered partition sums

$$
\begin{aligned}
P_{n}\left(x_{1}, x_{2}, \cdots, x_{n}\right) & =\sum_{X} A_{X} \delta(X) \\
p_{y_{1} y_{2} \cdots y_{n}} & =\sum_{Y} B_{Y} \delta(Y),
\end{aligned}
$$

where $A_{X}=D_{X} / Z, B_{X}=Z_{Y}^{*} / Z_{11 \cdots 1}^{*}$. Combining (7), (16) and using (46), the identities (49) and (50) now establish the conjecture of [5], which we now state as a

Theorem:

$$
\begin{aligned}
A_{X} & =q^{-|X|} B_{Y}, & & \text { for planar } X \leftrightarrow Y, \\
& =0, & & \text { otherwise. }
\end{aligned}
$$


Explicitly, the coefficients $B_{Y}$ are given in terms of $p_{y_{1} y_{2} \cdots y_{n}}$ by the Möbius inversion (41). Thus, the second line in (52) leads to identities relating partial partition functions $Z_{X}$. For example, from the second line of (44) and $A_{1212}=D_{1212}=0$, we obtain

$$
Z_{1212}-Z_{1214}-Z_{1232}+Z_{1234}=0
$$

This is the $n=4$ identity reported in [5]. Generally, one obtains an identity $A_{X}=0$ for each non-planar $X$. Using these equations one can express all non-planar $Z_{X}$ in terms of planar ones, a fact reached in [5] through the use of high-temperature expansions.

One can also write down the transformation relating $Z_{X}$ and $Z_{Y}^{*}$. For general $n$ and planar $X$ one finds by combining (40), (49) and (41),

$$
Z_{X}=C_{n}(q) \sum_{X^{\prime} \preceq X} q^{\left|Y^{\prime}\right|} \sum_{Y^{\prime \prime} \preceq Y^{\prime}} \mu\left(Y^{\prime \prime}, Y^{\prime}\right) Z_{Y^{\prime \prime}}^{*}, \quad X^{\prime} \leftrightarrow Y^{\prime} .
$$

The transformation relating $P_{n}\left(x_{1}, \cdots, x_{n}\right)$ to $p_{y_{1} \cdots y_{n}}$ can be written down similarly with the factor $C_{n}(q)$ replaced by $q^{-(n+1)}$. More explicitly, define a row vectors $\tilde{\mathbf{z}}_{n}$ and $\tilde{\mathbf{z}}_{n}^{*}$ whose elements are the $c_{n}$ partial partition functions corresponding to planar $X$, we find

$$
\mathbf{z}_{n}=q C_{n}(q) \mathbf{T}_{n}(q) \cdot \mathbf{z}_{n}^{*}
$$

where $\mathbf{T}_{n}(q)$ is a $c_{n} \times c_{n}$ matrix satisfying the identity

$$
\left[\mathbf{T}_{n}(q)\right]^{2}\left(X, X^{\prime}\right)=q^{n-1} \delta\left(x_{1}, x_{2}^{\prime}\right) \delta\left(x_{2}, x_{3}^{\prime}\right) \cdots \delta\left(x_{n}, x_{1}^{\prime}\right) .
$$

Note that the coefficient $q^{n-1}$ on the RHS differs from that in (21). Expressions of $\mathbf{T}_{n}(q)$ for $n=2,3$ have been given in (30) and (36). The expression for $n=4$ can be deduced from results reported in [5]. For $n=5$ there are 42 planar $X$ and we define the row vector

$$
\begin{aligned}
\tilde{\mathbf{z}}_{5} & =\left(Z_{11111},\left(Z_{11115}, Z_{11141}, Z_{11311}, Z_{12111}, Z_{12222}\right),\left(Z_{11144}, Z_{11331}, Z_{12211}, Z_{11333}, Z_{12221}\right)\right. \\
& \left(Z_{11145}, Z_{11341}, Z_{12311}, Z_{12333}, Z_{12225}\right),\left(Z_{11335}, Z_{12241}, Z_{11344}, Z_{12331}, Z_{12244}\right) \\
& \left(Z_{11315}, Z_{12141}, Z_{12311}, Z_{12115}, Z_{12242}\right),\left(Z_{11343}, Z_{12321}, Z_{12144}, Z_{12332}, Z_{12215}\right) \\
& \left(Z_{12125}, Z_{12142}, Z_{12312}, Z_{12313}, Z_{12323}\right),\left(Z_{11345}, Z_{12341}, Z_{12344}, Z_{12335}, Z_{12245}\right) \\
& \left.\left(Z_{12145}, Z_{12342}, Z_{12315}, Z_{12343}, Z_{12325}\right), Z_{12345}\right)
\end{aligned}
$$

Then after some algebras we deduce from (54) the explicit transformation

$$
\begin{aligned}
& Z_{11111}=\left\{1+q_{1}(1,1,1,1,1)+q_{1}(1,1,1,1,1)+q_{1} q_{2}(1,1,1,1,1)+q_{1} q_{2}(1,1,1,1,1)\right. \\
& \left.\quad+q_{1}^{2}(1,1,1,1,1)+q_{1}^{2}(1,1,1,1,1)+q_{1} s(1,1,1,1,1)+q_{1}^{2} q_{2}(1,1,1,1,1)+q_{1} q_{2} t\right\} \\
& \quad Z_{11115}=\left\{1+\left(-1, q_{1}, q_{1}, q_{1},-1\right)+\left(-1, q_{1}, q_{1},-1, q_{1}\right)-q_{2}\left(1,-q_{1},-q_{1}, 1,1\right)\right. \\
& \quad-q_{2}\left(1,-q_{1}, 1,-q_{1}, 1\right)-q_{1}\left(1,-q_{1}, 1,1,1\right)-q_{1}\left(1,-q_{1}, 1,1,1\right)+s\left(1,-q_{1}, 1,1,1\right) \\
& \left.\quad-q_{1} q_{2}(1,1,1,1,1)-q_{2} t\right\} \\
& \quad Z_{11144}=\left\{1+\left(q_{1},-1, q_{1}, q_{1},-1\right)-\left(1,1,-q_{1}, 1,1\right)-q_{2}\left(1,1,-q_{1}, 1,1\right)-q_{2}(1,1,1,1,1)\right. \\
& \quad+\left(-q_{1}^{2},-q_{1},-q_{1}, q_{1}^{2}, 1\right)+\left(1,-q_{1},-q_{1}, 1, q_{1}^{2}\right)-(p, s, s, p, p) \\
& \left.\quad+q_{2}\left(-q_{1}, 1, q_{1} q_{2}, 1,-q_{1}\right)-q_{2} u\right\}
\end{aligned}
$$




$$
\begin{aligned}
& Z_{11145}=\left\{1-\left(1,1,-q_{1},-q_{1}, 1\right)-\left(1,1,-q_{1}, 1,1\right)+\left(2,-q_{2}, q_{1} q_{2},-q_{2}, 2\right)-\left(-2, q_{2}, q_{2}, q_{2}, q_{2}\right)\right. \\
& \quad-\left(q_{1}, q_{1}, q_{1}, q_{1},-1\right)-\left(-1, q_{1}, q_{1},-1, q_{1}\right)+(h,-s,-s, h, h) \\
& \left.\quad+\left(2 q_{1}, q_{2},-q_{1} q_{2}, q_{2}, 2 q_{1}\right)+2(t+q)\right\} \\
& \quad Z_{11335}=\left\{1-\left(1,-q_{1}, 1,-q_{1}, 1\right)-(1,1,1,1,1)-q_{2}(1,1,1,1,1)+\left(2,-q_{2}, 2,-q_{2}, 2\right)\right. \\
& \left.\quad+\left(1, q_{1}^{2}, 1,-q_{1},-q_{1}\right)+\left(-q_{1}, 1,-q_{1}, 1,1\right)+(h,-p, h, h, h)-\left(q_{1} q_{2}, q_{2}, q_{2}, q_{1} q_{2}, 2\right)+2 u\right\}, \\
& Z_{11315}=\left\{1-\left(1,1,1,-q_{1}, 1\right)-\left(1,-q_{1}, 1,1,-q_{1}\right)-\left(-2, q_{2}, q_{2}, q_{2}, q_{2}\right)+\left(-q_{2},-q_{2}, 2, q_{1} q_{2}, 2\right)\right. \\
& \left.\quad+\left(1,-q_{1}, 1,-q_{1}, 1\right)+\left(1,-q_{1}, 1,-q_{1}, 1\right)+(e,-s, h,-s, e)+\left(2 q_{1}, q_{2}, 2 q_{1}, 2 q_{1}, q_{2}\right)+v\right\}, \\
& Z_{11343}=\left\{1-\left(1,1,1,-q_{1}, 1\right)-\left(-q_{1}, 1,1,1,1\right)-\left(q_{2},-2, q_{2}, q_{2},-2\right)-\left(-2,-2, q_{2}, q_{2}, q_{2}\right)\right. \\
& \left.\quad+\left(1,-q_{1}, 1,-q_{1}, 1\right)+(1,1,1,1,1)+(e, h,-p, h, e)-\left(q_{1} q_{2}, 2,-q_{2},-q_{2}, 2\right)+v+3 q\right\}, \\
& \quad Z_{11345}=\left\{1-\left(1,1,1,-q_{1}, 1\right)-(1,1,1,1,1)+\left(2,2,-q_{2},-q_{2}, 2\right)+\left(2,2,2,-q_{2}, 2\right)\right. \\
& \left.\quad+\left(1,-q_{1}, 1,-q_{1}, 1\right)+\left(1,1,-q_{1}, 1,1\right)+(-5, h, h, h,-5)+\left(2 q_{1},-2, q_{2}, q_{2},-2\right)+14-5 q\right\}, \\
& \quad Z_{12145}=\left\{1-(1,1,1,1,1)-\left(1,1,-q_{1}, 1,1\right)+\left(2,2,-q_{2}, 2,2\right)+\left(2,-q_{2}, 2,2,-q_{2}\right)\right. \\
& \left.\quad+(1,1,1,1,1)+\left(1,-q_{1}, 1,1,-q_{1}\right)-(q+5,5,5,5,-h)-\left(2,2,-q_{2}, 2,2\right)+14-2 q\right\},
\end{aligned}
$$

where $q_{m}=q-m, e=q-5, h=2 q-5, s=q^{2}-5 q+5, p=q^{2}-4 q+5, t=q^{2}-7 q+7, u=q^{2}-$ $5 q+7, v=q^{2}-10 q+14$. Here, one reads off elements of $\mathbf{T}_{5}(q)$ from (58) directly. For example, the second row of $\mathbf{T}_{5}(q)$ has elements $\left\{1,\left(-1, q_{1}, q_{1}, q_{1},-1\right),(-1, \cdots), \cdots,-q_{2} t\right\}$. Elements not shown are given by cyclic permutations of the partition indices within each set of parentheses. For example, the third row of $\mathbf{T}_{5}(q)$ is $\left\{1,\left(-1,-1, q_{1}, q_{1}, q_{1}\right),\left(q_{1}, \cdots\right), \cdots,-q_{2} t\right\}$, where elements within each set of parentheses are obtained by cyclically permuting those in the second row.

\section{THE MULTI-COMPONENT POTTS AND CHIRAL POTTS MODELS}

The chiral Potts model can be generalized to more-than-one components. An mcomponent chiral Potts model [7,8] is a

$$
Q=N_{1} N_{2} \cdots N_{m}
$$

state spin model, where $N_{\ell}, \ell=1,2, \cdots, m$, are positive integers. The interaction matrix $\mathbf{U}$ is a $Q \times Q$ matrix in the form of a direct product of $m$ cyclic matrices, with elements indexed by $U\left(x_{1}, \cdots, x_{m} \mid x_{1}^{\prime}, \cdots, x_{m}^{\prime}\right), x_{\ell}, x_{\ell}^{\prime}=1,2, \cdots, N_{\ell}, \ell=1, \cdots, m$, satisfying the cyclic property

$$
U\left(x_{1}, \cdots, x_{m} \mid x_{1}^{\prime}, \cdots, x_{m}^{\prime}\right)=u\left(x_{1}-x_{1}^{\prime}, \cdots, x_{m}-x_{m}^{\prime}\right)
$$

The $m=N_{1}=N_{2}=2$ version is known as the Ashkin-Teller model [9].

It is convenient to introduce vectors $\mathbf{x}=\left(x_{1}, \cdots, x_{m}\right)$ and $\mathbf{y}=\left(y_{1}, \cdots, y_{m}\right)$ and their scalar product

$$
\mathbf{x} \cdot \mathbf{y}=x_{1} y_{1} / N_{1}+\cdots+x_{m} y_{m} / N_{m}, \quad x_{\ell}, y_{\ell}=1, \cdots, N_{\ell}
$$


Then the dual model has the Boltzmann factor

$$
u^{*}(\mathbf{y})=\frac{1}{\sqrt{Q}} \sum_{x_{1}=1}^{N_{1}} \cdots \sum_{x_{m}=1}^{N_{m}} e^{2 \pi i \mathbf{x} \cdot \mathbf{y}} u(\mathbf{x})
$$

and the duality relation (7) now reads

$$
Q^{-N / 2} Z[u(\mathbf{x})]=Q^{-N^{*} / 2} Z^{(D)}\left[u^{*}(\mathbf{y})\right]
$$

Here, again, $\sqrt{Q} u^{*}(\mathbf{y})$ are the eigenvalues of the interaction matrix $\mathbf{U}$.

Proceeding in a similar fashion as before, we arrive at the following duality relation in place of $(13)$,

$$
Z_{\mathbf{x}_{1} \mathbf{x}_{2} \cdots \mathbf{x}_{n}}=C_{n}(Q) \sum_{\mathbf{y}_{1} \mathbf{y}_{2} \cdots \mathbf{y}_{n}} M\left(\mathbf{x}_{1}, \mathbf{x}_{2}, \cdots, \mathbf{x}_{n} \mid \mathbf{y}_{1}, \mathbf{y}_{2}, \cdots, \mathbf{y}_{n}\right) Z_{\mathbf{y}_{1} \mathbf{y}_{2} \cdots \mathbf{y}_{n}}^{*}
$$

Here, $\mathbf{M}$ is a $Q^{n} \times Q^{n}$ matrix with elements

$$
M\left(\mathbf{x}_{1}, \mathbf{x}_{2}, \cdots, \mathbf{x}_{n} \mid \mathbf{y}_{1}, \mathbf{y}_{2}, \cdots, \mathbf{y}_{n}\right)=\exp \left[2 \pi i\left(\mathbf{x}_{n 1} \cdot \mathbf{y}_{1}+\mathbf{x}_{12} \cdot \mathbf{y}_{2}+\cdots+\mathbf{x}_{(n-1) n} \cdot \mathbf{y}_{n}\right)\right]
$$

satisfying the reciprocal inversion relation

$$
\mathbf{M}^{2}\left(\mathbf{x}_{1}, \mathbf{x}_{2}, \cdots, \mathbf{x}_{n} \mid \mathbf{x}_{1}^{\prime}, \mathbf{x}_{2}^{\prime}, \cdots, \mathbf{x}_{n}^{\prime}\right)=Q^{n+1} \delta\left(\mathbf{x}_{1}, \mathbf{x}_{2}^{\prime}\right) \delta\left(\mathbf{x}_{2}, \mathbf{x}_{3}^{\prime}\right) \cdots \delta\left(\mathbf{x}_{n}, \mathbf{x}_{1}^{\prime}\right)
$$

Defining the $n$-point correlation functions

$$
\begin{aligned}
P_{n}\left(\mathbf{x}_{1}, \mathbf{x}_{2}, \cdots, \mathbf{x}_{n}\right) & =Z_{\mathbf{x}_{1} \mathbf{x}_{2} \cdots \mathbf{x}_{n}} / Z(u) \\
p_{\mathbf{y}_{1} \mathbf{y}_{2} \cdots \mathbf{y}_{n}} & =Q Z_{\mathbf{y}_{1} \mathbf{y}_{2} \cdots \mathbf{y}_{n}}^{*} / Z^{(D)}\left(u^{*}\right)
\end{aligned}
$$

then (64) becomes

$$
P_{n}\left(\mathbf{x}_{1}, \mathbf{x}_{2}, \cdots, \mathbf{x}_{n}\right)=Q^{-(n+1)} \sum_{\mathbf{y}_{1} \mathbf{y}_{2} \cdots \mathbf{y}_{n}} M\left(\mathbf{x}_{1}, \mathbf{x}_{2}, \cdots, \mathbf{x}_{n} \mid \mathbf{y}_{1}, \mathbf{y}_{2}, \cdots, \mathbf{y}_{n}\right) p_{\mathbf{y}_{1} \mathbf{y}_{2} \cdots \mathbf{y}_{n}}
$$

This is the most general correlation duality relation for the $m$-component chiral Potts model. An immediate consequence of (68) is, in analogous to (11) and (18), the following expression for the $n$-point correlation

$$
\Gamma_{n} \equiv Q^{n} P_{n}(\mathbf{x}, \mathbf{x}, \cdots, \mathbf{x})-1=Q^{-1} \sum_{\mathbf{y}_{1} \mathbf{y}_{2} \cdots \mathbf{y}_{n}} p_{\mathbf{y}_{1} \mathbf{y}_{2} \cdots \mathbf{y}_{n}}-1
$$

The m-component Potts model: The $m$-component chiral Potts model reduces to an $m$ component (standard) Potts model when the interaction (60) assumes the form (5) for each component, namely,

$$
u\left(x_{1}, \cdots, x_{m}\right)=\prod_{\ell=1}^{m}\left[1+\left(e^{K_{\ell}}-1\right) \delta_{N_{\ell}}\left(x_{\ell}, 0\right)\right], \quad x_{\ell}=1,2, \cdots, N_{\ell},
$$

where 


$$
\begin{aligned}
\delta_{N_{\ell}}\left(x, x^{\prime}\right) & =1, & & x=x^{\prime} \quad\left(\bmod N_{\ell}\right), \\
& =0, & & x \neq x^{\prime}
\end{aligned}
$$

The $m=2$ version is known as the $\left(N_{1}, N_{2}\right)$ model [10].

Now each $\mathbf{x}_{i}$ in (68) is an $m$-component vector. Let its components be $x_{i \ell}=1, \cdots, N_{\ell}, i=$ $1, \cdots, n ; \ell=1, \cdots, m$, and let $X_{\ell}$ denote the partition of the $n$ integers $1,2, \cdots, n$ dictated by $\left\{x_{i \ell}, i=1, \cdots, n\right\}$. Then, in analogous to (51), we write the partially ordered mappings

$$
\begin{aligned}
P_{n}\left(\mathbf{x}_{1}, \mathbf{x}_{2}, \cdots, \mathbf{x}_{n}\right) & =\sum_{X_{\ell}} A_{X_{1} X_{2} \cdots X_{m}} \delta_{N_{1}}\left(X_{1}\right) \delta_{N_{2}}\left(X_{2}\right) \cdots \delta_{N_{m}}\left(X_{m}\right), \\
p_{\mathbf{y}_{1} \mathbf{y}_{2} \cdots \mathbf{y}_{n}} & =\sum_{Y_{\ell}} B_{Y_{1} Y_{2} \cdots Y_{m}} \delta_{N_{1}}\left(Y_{1}\right) \delta_{N_{2}}\left(Y_{2}\right) \cdots \delta_{N_{m}}\left(Y_{m}\right) .
\end{aligned}
$$

With these notations, we have the following corollary of our Theorem:

\section{Corollary:}

For the $m$-component Potts model whose correlation functions are (72), we have

$$
\begin{aligned}
A_{X_{1} X_{2} \cdots X_{m}} & =\prod_{\ell=1}^{m} N_{\ell}^{-\left|X_{\ell}\right|} B_{Y_{1} Y_{2} \cdots Y_{m}}, & & \text { for planar } X_{\ell} \leftrightarrow Y_{\ell}, \\
& =0, & & \text { otherwise. }
\end{aligned}
$$

Here, explicitly, by using

$$
\begin{aligned}
Z_{X_{1} X_{2} \cdots X_{m}} / Z & =\sum_{X_{\ell}^{\prime} \preceq X_{\ell}} A_{X_{1}^{\prime} X_{2}^{\prime} \cdots X_{m}^{\prime}} \\
B_{Y_{1} Y_{2} \cdots Y_{m}} & =\sum_{Y_{\ell}^{\prime} \preceq Y_{\ell}} p_{Y_{1}^{\prime} Y_{2}^{\prime} \cdots Y_{m}^{\prime}} \prod_{\ell=1}^{m} \mu\left(Y_{\ell}^{\prime}, Y_{\ell}\right),
\end{aligned}
$$

in analogous to (54) one can reduce the correlation duality relation (64) to

$$
\begin{aligned}
Z_{X_{1} X_{2} \cdots X_{m}}=C_{n}(Q) & \sum_{X_{\ell}^{\prime} \preceq X_{\ell}} \sum_{Y_{\ell}^{\prime \prime} \preceq Y_{\ell}^{\prime}} Z_{Y_{1}^{\prime \prime} Y_{2}^{\prime \prime} \cdots Y_{m}^{\prime \prime}}^{m} \\
\times & \prod_{\ell=1}^{m} N_{\ell}^{\left|Y_{\ell}^{\prime}\right|} \mu\left(Y_{\ell}^{\prime \prime}, Y_{\ell}^{\prime}\right), \quad X_{\ell}^{\prime} \leftrightarrow Y_{\ell}^{\prime} .
\end{aligned}
$$

This is the desired duality relation for the $m$-component Potts model. Now there are $\left(c_{n}\right)^{m}$ partial partition functions $Z$ and $Z^{*}$. Considering $Z$ and $Z^{*}$ as tensors $\mathbf{Z}_{m}$ and $\mathbf{Z}_{m}^{*}$ of rank $m$, one can rewrite (75) more compactly as

$$
\mathbf{Z}_{m}=Q C_{n}(Q)\left[\mathbf{T}_{n}\left(N_{1}\right) \otimes \cdots \otimes \mathbf{T}_{n}\left(N_{m}\right)\right] \cdot \mathbf{Z}_{m}^{*}
$$

where the tensor products are over $m c_{n} \times c_{n}$ matrices $\mathbf{T}_{n}(q)$ defined in (55). The last expression generalizes the $m=2$ results for the $\left(N_{1}, N_{2}\right)$ model reported in [11. 


\section{SUMMARY AND ACKNOWLEDGEMENT}

We have considered the chiral Potts model and obtained the duality relation for its correlation functions of $n$ sites on the boundary of a planar lattice. The result is given by (13) and (17). By specializing this result to the standard Potts model, we establish a recent conjecture of [5] on the correlation duality which we now state in (52) as a Theorem. The explicit duality relation relating the partial partition functions is given in (54), with the $n=2,3,5$ expressions explicitly worked out. The formulation is next extended to the multicomponent chiral Potts model, leading to the correlation duality relations (64) and (68). Again, specializing the results to the $m$-component Potts model, we obtain the Corollary (73) and the correlation duality relations (75) and (76).

Work has been supported in part by NSF Grant DMR-9614170. 


\section{REFERENCES}

[1] Potts R B 1952 Proc. Camb. Phil. Soc. 48106

[2] Wu F Y 1982 Rev. Mod. Phys. 54235

[3] Wu F Y and Wang Y K 1976 J. Math. Phys. 17439

[4] Wu F Y 1997 Phys. Lett. A 22843

[5] Wu F Y and Huang H Y 1997 Phys. Rev. Lett. to appear

[6] Au-Yang H, McCoy B M, Perk J H H, Tang S and Yan M-L 1987 Phys. Lett. A 123 219

[7] Au-Yang H and Perk J H H 1991 Physica A 177139

[8] Au-Yang H and Perk J H H 1991 Int. J. Mod. Phys. A 7 Suppl 1B 1007, 1025

[9] Ashkin J and Teller E 1943 Phys. Rev. 64178

[10] Domany E and Riedel E K 1978 Phys. Rev. Lett. 40561

[11] Wu F Y and Lu W T 1998 Ch. J. Phys. 36 to appear

[12] Jacobsen J L 1997 Phys. Lett. A 233489

[13] van Lint L J and Wilson R M 1992 A Course in Combinatorics (Cambridge University Press), p. 301

[14] Temperley H N V and Lieb E H 1971 Proc. Roy. Lond. A 322251

[15] Blöte H W J and Nightingale M P 1982 Physica A 112405 


\section{Figure captions}

Fig. 1. A planar graph $\mathcal{L}$ and $n$ sites $i, j, \ldots m, \ell$ on the boundary.

Fig. 2. Reciprocal graphs $\mathcal{L}$ (solid lines) and $\mathcal{L}^{*}$ (broken lines).

Fig. 3. Graphical representation $G_{n}$ of the matrix element (65).

Fig. 4. Examples of connectivities on $G_{4}$. (a) $X=\{1133\}$. (b) $X=\{1114\}$. (c) $X=$ $\{1212\}$.

Fig. 5. The contraction of the the $Y=\{123216\}$ graph of $G_{6}$ into a cactus tree. 


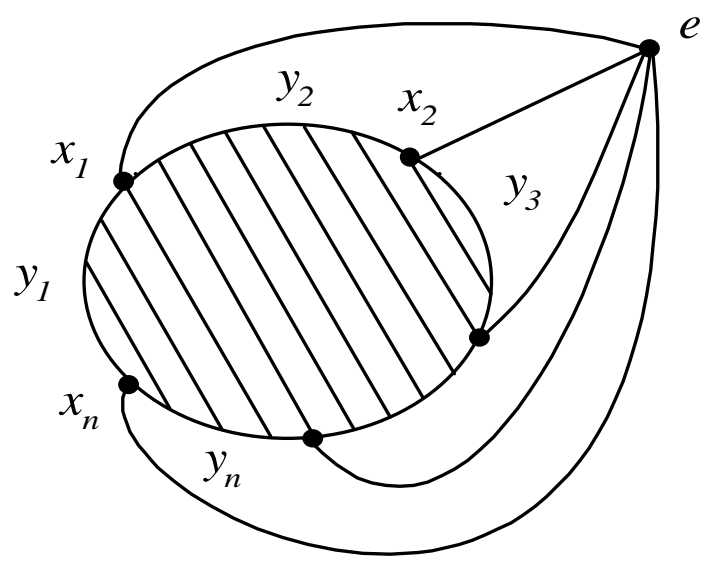

Fig. 1 


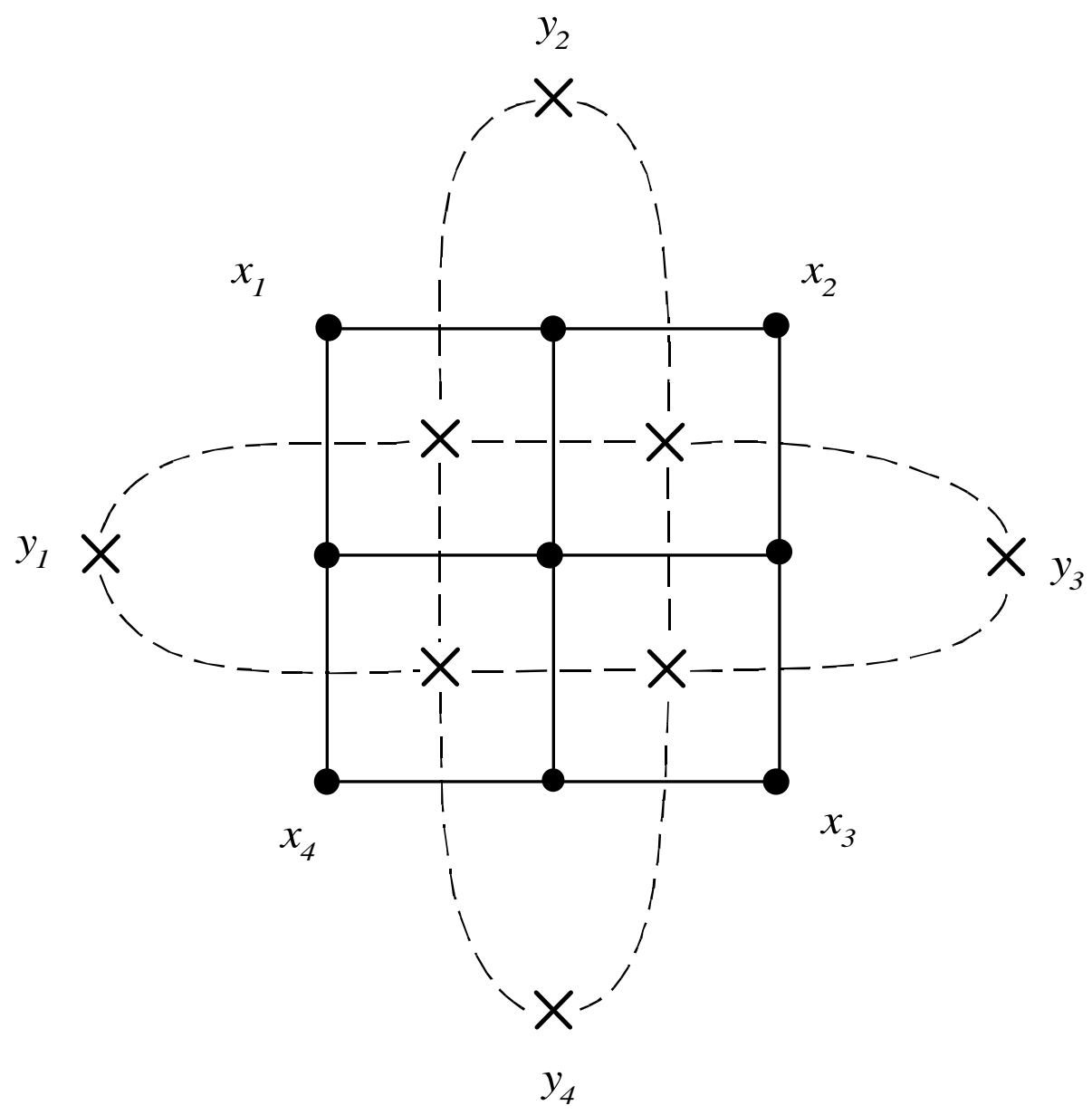

Fig. 2 


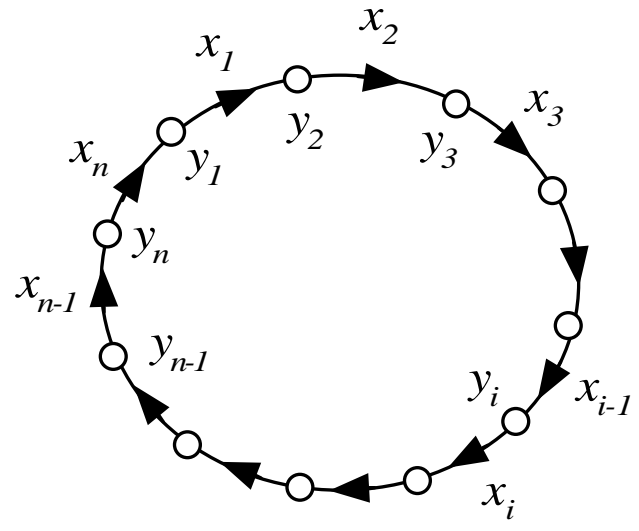

Fig. 3 


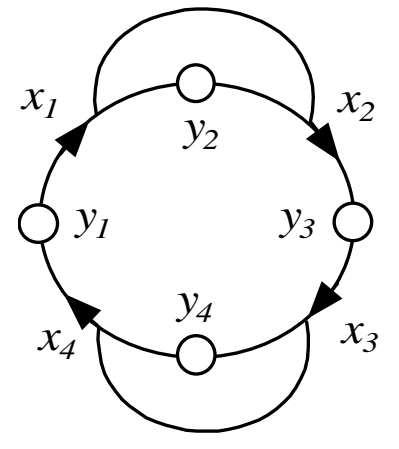

(a)
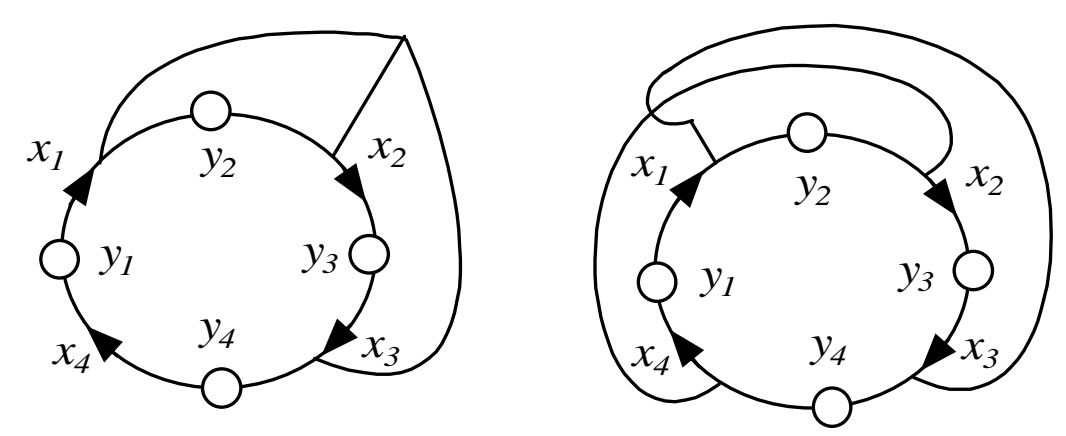

Fig. 4

(c) 


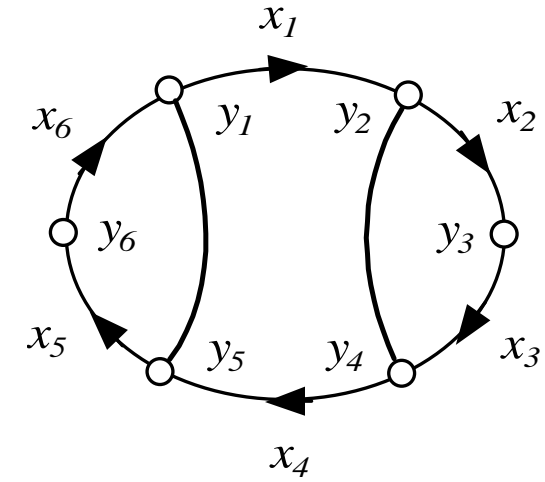

(a)

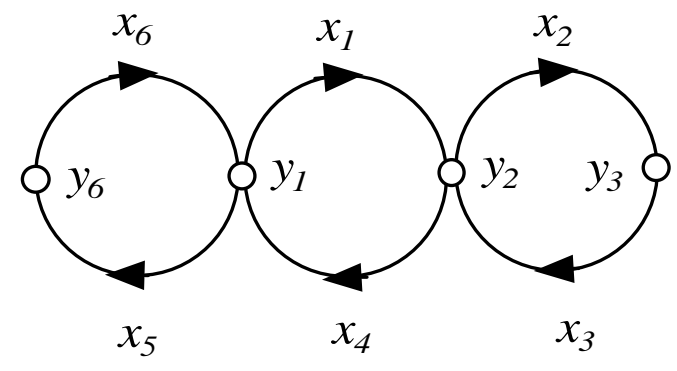

(b)

Fig. 5 